\title{
Dynamics Modeling and Validation of a 3-PRR Planar Parallel Robot
}

\author{
$1^{\text {st }}$ Qi Sun \\ Department of Mechanical Engineering \\ and Centre for Intelligent Machines \\ McGill University \\ Montreal, Quebec \\ sunqi@cim.mcgill.ca
}

\author{
$2^{\text {nd }}$ Jorge Angeles \\ Department of Mechanical Engineering \\ and Centre for Intelligent Machines \\ McGill University \\ Montreal, Quebec \\ angeles@cim.mcgill.ca
}

\author{
$3^{\text {rd }}$ James Richard Forbes \\ Department of Mechanical Engineering \\ and Centre for Intelligent Machines \\ McGill University \\ Montreal, Quebec \\ jforbes@cim.mcgill.ca
}

\begin{abstract}
A systematic and effective approach to the formulation of the dynamics of a planar parallel robot is proposed in this paper, based on the concept of the natural orthogonal complement (NOC). This approach is demonstrated on a robot designed for high-frequency, small-amplitude operations. Its kinematic relations are formulated based on planar screw theory. Then, the constraint wrenches in the Newton-Euler equations are eliminated with the aid of the NOC, namely, the twist-shaping matrix, which maps the active joint-rate array into the robot twist array. The dynamics model of minimum dimension is formulated. Then, upon comparison with the ADAMS simulation results, our model is proven to be not only accurate, but also effective for the dynamics modeling, which shows that the approach is suitable for the dynamics simulation and real-time control of the robot of interest.
\end{abstract}

Index Terms-dynamics modeling, parallel robot, planar, natural orthogonal complement, validation

\section{INTRODUCTION}

For a parallel-kinematics machine (PKM), the dynamics model becomes crucial not only for the system simulation, but also for its effective model-based control. This model describes the relations among the position, velocity and acceleration of the active joints and the actuator torques. Dynamics models of multi-body systems are generally derived based on: the Newton-Euler formulation [1], the Euler-Lagrange formulation [2], the principle of virtual work [3], Hamilton's principle [4] or Kane's equations [5]. All the methods mentioned above have their own advantages and disadvantages.

The Newton-Euler formulation is classical, but still present in dynamics modeling. In this method, the Newton-Euler equations are formulated based on the free-body diagram. Elimination of the nonworking constraint wrenches is the key step in the derivation of the minimal-order constrained dynamics equations of the system. The concept of the natural orthogonal complement (NOC) was first introduced by Angeles and Lee [6] as the tool to eliminate the constraint wrenches. The NOC is defined as the transformation matrix that maps the independent joint rates into the system twist. It becomes naturally an orthogonal complement of the system velocity constrains. The efficiency of the NOC has been shown on its application to the dynamics modeling of serial mechanical systems [7]. However, for PKMs, due to the inherent complexity caused by their closed-loop structure, the derivation of the NOC poses its own challenges.

The subject of this paper is the formulation of the dynamics of a PKM based on the NOC. This approach is applied and validated on a planar parallel robot designed for highfrequency, small-amplitude operations. In Section 2, the robot architecture is introduced. The kinematic relations of each limb chain and the kinematic relations between the activejoint rates and the Cartesian twist are formulated based on the screw theory, as applied to planar systems in Section 3. Based on the foregoing kinematic relations, the NOC of the robot is first derived, then its dynamics in Section 4. In Section 5 , upon comparison of the simulation results obtained with ADAMS, the dynamics model in question is validated. Finally, conclusions are the subject of Section 6.

\section{Robot ARCHItecture}

The subject of this paper is the dynamics modeling of a 3 - PRR $^{1}$ planar parallel robot, whose architecture is shown in Fig. 1, and the model validation. This robot bears a symmetric architecture. The moving platform (MP) and the base platform (BP), defining equilateral triangles, are connected via three identical limbs. Each limb is a serial PRR chain, with the prismatic actuator mounted on the fixed base. This robot is designed to generate high-frequency, small-amplitude vibrations in the plane, for applications such as rigid-body inertia-parameter identification, earthquake simulation, and so on. The axes of the $\mathrm{R}$ joints are normal to the plane of motion, while the directions of the $\underline{\mathrm{P}}$ joints are coplanar. The centers $\left\{A_{i}\right\}_{1}^{3}$ of the $\mathrm{R}$ joints carried by the prismatic actuators trace corresponding sides of an equilateral triangle of vertices $\left\{B_{i}\right\}_{1}^{3}$. The centers of the proximal (to the BP) $\mathrm{R}$ joints are $\left\{A_{i}\right\}_{1}^{3}$, those of the distal $\mathrm{R}$ joints being $\left\{M_{i}\right\}_{1}^{3}$. The MP plane is that of the equilateral triangle with vertices $\left\{M_{i}\right\}_{1}^{3}$. The centroid $C$ of the MP triangle is selected as the operation point. The robot under study is a generator of the planarmotion subgroup [8].

For the purpose of analysis, a Cartesian frame $O X Y Z$ is attached to the BP, with its origin located at the centroid $O$

\footnotetext{
${ }^{1} \mathrm{P}$ and $\mathrm{R}$ stand for prismatic and revolute joints, respectively. Moreover, actuated joints are underlined.
} 
of the BP triangle. Moreover, the $X$-axis points in the $B_{3} B_{1}$ direction; the $Y$-axis points along the $O B_{2}$ direction; and the $Z$-axis is normal to the $\mathrm{BP}$ and passes through $O$.

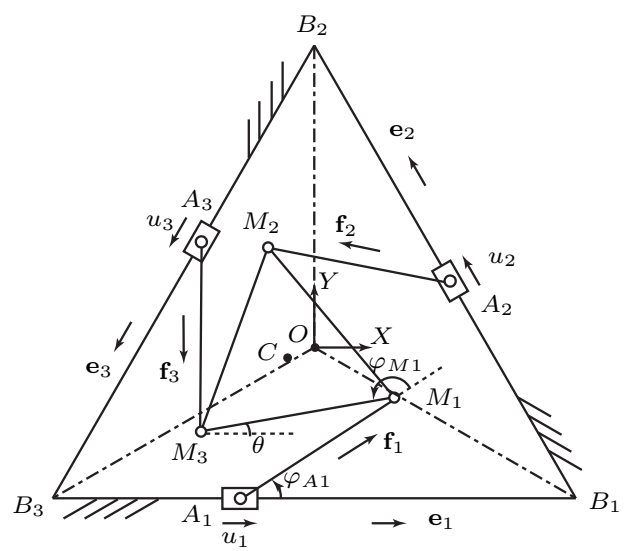

Fig. 1. An arbitrary posture of the 3-PRR planar parallel robot

\section{KINEMATICS}

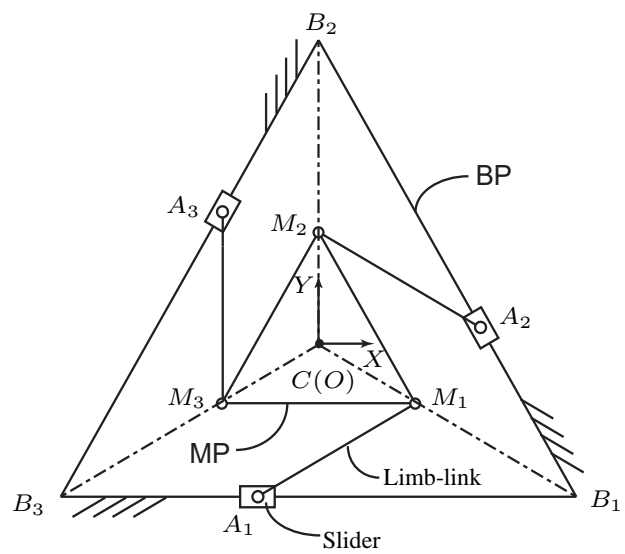

Fig. 2. Reference posture of the 3-PRR planar parallel robot

The reference posture of the robot is illustrated in Fig. 2. At this posture, the sides of the MP triangle are parallel to the corresponding sides of the BP triangle, while the centroid $C$ of the MP triangle coincides with the origin $O$ of the inertia frame $\{X, Y, Z\}$. The arbitrary posture shown in Fig. 1 is defined by the pose of the MP, which is represented by a threedimensional array $\mathbf{p}$, the planar screw of the MP, namely,

$$
\mathbf{p}=\left[\begin{array}{l}
\theta \\
\mathbf{c}
\end{array}\right] \in \mathbb{R}^{3}, \quad \mathbf{c}=\left[\begin{array}{l}
x_{c} \\
y_{c}
\end{array}\right]
$$

where $\theta \in \mathbb{R}$ denotes the angle of rotation of the MP from its reference orientation, while $\mathbf{c}$ the position vector of the MP centroid $C$, represented by $\left[x_{c}, y_{c}\right]^{T}$, in the $O X Y$ plane. Then, the planar MP twist $\mathbf{t}_{M}$ is simply ${ }^{2}$ the time-derivative

\footnotetext{
${ }^{2}$ The relation $\omega=\dot{\theta}$ holds only in the planar case, not so in the threedimensional case!
}

of p, namely,

$$
\mathbf{t}_{M}=\dot{\mathbf{p}}=\left[\begin{array}{c}
\dot{\theta} \\
\dot{\mathbf{c}}
\end{array}\right]=\left[\begin{array}{c}
\omega \\
\dot{\mathbf{c}}
\end{array}\right]
$$

where $\omega$ denotes the MP angular velocity around the $Z$-axis, $\dot{\mathbf{c}}$ the velocity of the MP center of mass (c.o.m.), which coincides with its centroid.

The robot under study has three limbs, each being a serial PRR chain. For the $j$ th limb, $j=1,2,3$, the MP twist can be represented as a linear transformation of the array of rates of the $\underline{\mathrm{P}}$ joint and the two $\mathrm{R}$ joints, namely,

$$
\mathbf{t}_{M}=\mathbf{J}_{j} \dot{\gamma}_{j}, \quad j=1,2,3
$$

with

$$
\begin{gathered}
\mathbf{J}_{j}=\left[\begin{array}{ccc}
0 & 1 & 1 \\
\mathbf{e}_{j} & \mathbf{E p}_{A j} & \mathbf{E p}_{M j}
\end{array}\right] \in \mathbb{R}^{3 \times 3}, \quad \mathbf{E}=\left[\begin{array}{cc}
0 & -1 \\
1 & 0
\end{array}\right] \\
\dot{\gamma}_{j}=\left[\begin{array}{lll}
\dot{u}_{j} & \dot{\varphi}_{A j} & \dot{\varphi}_{M j}
\end{array}\right]^{T} \in \mathbb{R}^{3}
\end{gathered}
$$

where matrix $\mathbf{E}$ represents a rotation about the $Z$-axis through an angle of $90^{\circ}$. In Fig. 1 , the unit vectors $\mathbf{e}_{j} \in \mathbb{R}^{2}$ and $\mathbf{f}_{j} \in \mathbb{R}^{2}$ represent the directions of the $j$ th $\underline{\mathrm{P}}$ joint and of vector $\overrightarrow{A_{j} M_{j}}$, respectively. Moreover, $u_{j}$ denotes the displacement of the $j$ th $\underline{\mathrm{P}}$ joint from its reference configuration in Fig. 2. Furthermore, $\varphi_{A j}$ denotes the angle from $\mathbf{e}_{j}$ to $\overrightarrow{A_{j} M_{j}}$, and $\varphi_{M j}$ the angle from $\overrightarrow{A_{j} M_{j}}$ to the corresponding side of the MP triangle. Furthermore, $\mathbf{J}_{j}$ is the Jacobian mapping the joint-rate array $\dot{\gamma}_{j}$ of the $j$ th limb into the MP twist $\mathbf{t}_{M}$. The columns of $\mathbf{J}_{j}$ are the planar Plücker arrays of the joint axes in the $j$ th limb. Moreover, $\mathbf{p}_{M j}$ denotes vector $\overrightarrow{M_{j} C}$, while $\mathbf{p}_{A j}$ represents vector $\overrightarrow{A_{j} C}$.

Eliminating the passive joint rates, $\left\{\dot{\varphi}_{A j}\right\}_{1}^{3}$ and $\left\{\dot{\varphi}_{M j}\right\}_{1}^{3}$ in (3), the kinematic relation between the active joint-rate array $\dot{\mathbf{u}}=\left[\dot{u}_{1}, \dot{u}_{2}, \dot{u}_{3}\right]^{T}$ and the MP twist $\mathbf{t}_{M}$ is obtained as

$$
\mathbf{H} \mathbf{t}_{M}=\mathbf{D} \dot{\mathbf{u}}
$$

where $u_{j}, j=1,2,3$ denotes the speed of the $j$ th actuator. Moreover, $\mathbf{H}$ and $\mathbf{D}$ denote, respectively, the forward and the inverse $^{3}$ Jacobian matrices of the robot, relating the Cartesian velocity array $\dot{\mathbf{p}}$ with the active joint-rate array $\dot{\mathbf{u}}$. Matrix $\mathbf{D}$ is given below:

$$
\mathbf{D}=\operatorname{diag}\left(d_{1}, d_{2}, d_{3}\right), \quad d_{j}=\cos \varphi_{A j}, \quad j=1,2,3
$$

Moreover, the entries $h_{j, i}$ of matrix $\mathbf{H}$ are

$$
\begin{aligned}
& h_{j 1}=r \sin \left(\varphi_{M j}-\frac{\pi}{6}\right) \\
& h_{j 2}=\cos \left(\varphi_{A j}+\phi_{j}\right) \quad, \quad j=1,2,3 \\
& h_{j 3}=\sin \left(\varphi_{A j}+\phi_{j}\right)
\end{aligned}
$$

where $r$ is the circumradius of the MP triangle. Moreover, $\phi_{j}$ denotes the angle from the $X$-axis to $\mathbf{e}_{j}$, namely,

$$
\phi_{1}=0, \quad \phi_{2}=2 \pi / 3, \quad \phi_{3}=-2 \pi / 3
$$

${ }^{3}$ From the context, the qualifier does not mean a matrix inverse! 


\section{DYNAMICS}

\section{A. Dynamics of Each Link}

In following the established practice, the robot under study is modeled as a multi-rigid-body system. The seven moving rigid links are the three moving sliders, the three limb-links and the MP.

In the planar case, the inertia dyad ${ }^{4}$ of each moving rigid body is a $3 \times 3$ matrix, namely,

$$
\mathbf{M}_{i}=\left[\begin{array}{cc}
I_{i} & \mathbf{0}^{T} \\
\mathbf{0} & m_{i} \mathbf{1}
\end{array}\right], \quad i=1,2, \cdots, 7
$$

where $I_{i}$ represents the moment of inertia of the $i$ th moving rigid body around the $Z$-axis at its c.o.m., and $m_{i}$ the mass of the same body. Moreover, $\mathbf{0}$ is the two-dimensional zero vector, while $\mathbf{1}$ is the $2 \times 2$ identity matrix. The twist of the $i$ th moving rigid body is also a three-dimensional array, namely,

$$
\mathbf{t}_{i}=\left[\begin{array}{ll}
\omega_{i} & \dot{\mathbf{c}}_{i}^{T}
\end{array}\right]^{T}, \quad i=1,2, \cdots, 7
$$

with $\omega_{i}$ denoting the angular rate of the $i$ th moving rigid body, positive in the c.c.w. direction, and $\dot{\mathbf{c}}_{i}$ the velocity of the c.o.m. of the same body. The planar wrench $\mathbf{w}_{i}$ applied onto the $i$ th moving body is represented as

$$
\mathbf{w}_{i}=\mathbf{w}_{i}^{W}+\mathbf{w}_{i}^{C}=\left[\begin{array}{c}
n_{i} \\
\mathbf{f}_{i}
\end{array}\right], \quad i=1,2, \cdots, 7
$$

where $n_{i}$ and $\mathbf{f}_{i}$ denote the resultant moment about and the resultant force acting at the c.o.m. of the $i$ th moving body. Moreover, $\mathbf{w}_{i}^{W}$ represents the working wrench exerted on the $i$ th body by the environment and the actuators, while $\mathbf{w}_{i}^{C}$ the nonworking constraint wrench exerted, in the same body, by the neighbouring links.

Then, neglecting all the dissipative wrenches, the NewtonEuler equation of the $i$ th moving rigid body is expressed as

$$
\mathbf{M}_{i} \dot{\mathbf{t}}_{i}=\mathbf{w}_{i}^{A}+\mathbf{w}_{i}^{C}, \quad i=1,2, \cdots, 7
$$

with $\mathbf{w}_{i}^{A}$ denoting the actuator-supplied wrench, i.e., the active component of the working wrench $\mathbf{w}_{i}^{W}$, exerted on the $i$ th body. For the $j$ th limb, $j=1,2,3$, let $\mathbf{t}_{j}$ represent the twist of the slider and $\mathbf{t}_{j+3}$ that of the limb-link $A_{j} M_{j}$. Moreover, $\mathbf{t}_{7}=\mathbf{t}_{M}$ represents the MP twist.

\section{B. Dynamics of the Robot}

Combining the Newton-Euler equations of each moving rigid body, the dynamics of the overall robot is described by 21 uncoupled equations:

$$
\mathbf{M} \dot{\mathbf{t}}=\mathbf{w}^{A}+\mathbf{w}^{C}
$$

with

$$
\mathbf{M}=\operatorname{diag}\left(\mathbf{M}_{1}, \mathbf{M}_{2}, \cdots, \mathbf{M}_{7}\right) \in \mathbb{R}^{21 \times 21}
$$

\footnotetext{
${ }^{4}$ Here we borrow the term in italics from the original concept proposed by von Mises [9], as a $6 \times 6$ array, in the context of rigid-body dynamics in three-dimensional space.
}

$$
\mathbf{t}=\left[\begin{array}{c}
\mathbf{t}_{1} \\
\mathbf{t}_{2} \\
\vdots \\
\mathbf{t}_{7}
\end{array}\right], \quad \mathbf{w}^{A}=\left[\begin{array}{c}
\mathbf{w}_{1}^{A} \\
\mathbf{w}_{2}^{A} \\
\vdots \\
\mathbf{w}_{7}^{A}
\end{array}\right], \quad \mathbf{w}^{C}=\left[\begin{array}{c}
\mathbf{w}_{1}^{C} \\
\mathbf{w}_{2}^{C} \\
\vdots \\
\mathbf{w}_{7}^{C}
\end{array}\right] \in \mathbb{R}^{21}
$$

The next step is the elimination of the constraint-wrench vector array $\mathbf{w}^{C}$, followed by the reduction of the uncoupled system of 21 equations into a set of three coupled equations of motion. The kinematic constraint equations are expressed in a linear homogenous form in the robot-twist vector $\mathbf{t} \in \mathbb{R}^{21}$, namely,

$$
\mathbf{K} \mathbf{t}=\mathbf{0}, \quad \mathbf{t} \in \mathbb{R}^{21}, \quad \mathbf{K} \in \mathbb{R}^{21 \times 21}
$$

Moreover, the robot twist is determined by the speed of the actuators; therefore the twist vector $\mathbf{t}$ is expressed as a linear transformation of the active joint-rate array $\dot{\mathbf{u}}$, namely,

$$
\mathbf{t}=\mathbf{T} \dot{\mathbf{u}}, \quad \mathbf{T} \in \mathbb{R}^{21 \times 3}
$$

with $\mathbf{T}$ defined as the robot twist-shaping matrix.

Upon substitution of (15) into (16), we have

$$
\mathbf{K T} \dot{\mathbf{u}}=\mathbf{0}, \quad \forall \dot{\mathbf{u}} \in \mathbb{R}^{3}
$$

which shows that the product $\mathbf{K T}$ vanishes:

$$
\mathbf{K T}=\mathbf{O}_{21 \times 3}
$$

with $\mathbf{O}_{21 \times 3}$ denoting the $21 \times 3$ zero matrix. The robot twistshaping matrix $\mathbf{T}$ is referred to as the natural orthogonal complement (NOC) of the the constraint matrix $\mathbf{K}$.

The robot constraint wrench $\mathbf{w}^{C}$ produces no power on the system, i.e.,

$$
\mathbf{t}^{T} \mathbf{w}^{C}=\dot{\mathbf{u}}^{T} \mathbf{T}^{T} \mathbf{w}^{C}=0 \Rightarrow \mathbf{T}^{T} \mathbf{w}^{C}=\mathbf{0}
$$

The above equation shows that the transpose of the twistshaping matrix $\mathbf{T}$ is the wrench annihilator ${ }^{5}$ of $\mathbf{w}^{C}$.

Upon pre-multiplying (13) by $\mathbf{T}^{T}$ and substitution of (16) into (13), the joint-space dynamics model of the robot without constraint wrenches is obtained:

$$
\tau=\mathbf{I} \ddot{\mathbf{u}}+\mathbf{C} \dot{\mathbf{u}}
$$

with

$$
\mathbf{I} \equiv \mathbf{T}^{T} \mathbf{M T}, \quad \mathbf{C} \equiv \mathbf{T}^{T} \mathbf{M} \dot{\mathbf{T}}, \quad \tau \equiv \mathbf{T}^{T} \mathbf{w}^{A}
$$

where $\mathbf{I} \in \mathbb{R}^{3 \times 3}$ denotes the inertia matrix of the overall robot in joint space, and $\mathbf{C} \in \mathbb{R}^{3 \times 3}$ the matrix of Coriolis and centrifugal forces of the robot in the same space. Moreover, $\boldsymbol{\tau}=\left[\tau_{1}, \tau_{2}, \tau_{3}\right]^{T}$ represents the force array of the actuators, with $\tau_{j}, j=1,2,3$, denoting the force exerted by the $j$ th actuator onto its slider. The robot inertia matrix $\mathbf{I}$ in joint space is symmetric, positive-definite and posture-dependent. Moreover, it is dimensionally-homogenous, bearing units of $\mathrm{kg}$.

\footnotetext{
${ }^{5} \mathrm{~A}$ wrench annihilator is defined a matrix that maps a wrench vector array into the zero array [10].
} 
Further, matrix $\mathbf{T}$ is partitioned in the form

$$
\mathbf{T}=\left[\begin{array}{c}
\mathbf{T}_{1} \\
\mathbf{T}_{\mathbf{2}} \\
\vdots \\
\mathbf{T}_{7}
\end{array}\right], \quad \mathbf{T}_{i}=\frac{\partial \mathbf{t}_{i}}{\partial \dot{\mathbf{u}}}, \quad i=1,2, \cdots, 7
$$

with $\mathbf{T}_{i}$ denoting the twist-shaping matrix of the $i$ the body, mapping the actuator-speed array $\dot{\mathbf{u}}$ into the twist $\mathbf{t}_{i}$ of the same body. Therefore, $\mathbf{I}$ and $\mathbf{C}$ in (20) become

$$
\mathbf{I}=\sum_{i=1}^{7} \mathbf{T}_{i}^{T} \mathbf{M}_{i} \mathbf{T}_{i}, \quad \mathbf{C}=\sum_{i=1}^{7} \mathbf{T}_{i}^{T} \mathbf{M}_{i} \dot{\mathbf{T}}_{i}
$$

The twist $\mathbf{t}_{j}$ of the $j$ th slider is readily obtained:

$$
\mathbf{t}_{j}=\dot{u}_{j}\left[\begin{array}{c}
0 \\
\mathbf{e}_{j}
\end{array}\right] \in \mathbb{R}^{3}, \quad \mathbf{e}_{j} \in \mathbb{R}^{2}, \quad j=1,2,3
$$

Therefore, the twist-shaping matrices $\mathbf{T}_{j} \in \mathbb{R}^{3 \times 3}, j=1,2,3$ of the sliders are, with $\mathbf{0} \in \mathbb{R}^{2}$,

$$
\begin{aligned}
& \mathbf{T}_{1}=\left[\begin{array}{lll}
0 & 0 & 0 \\
\mathbf{e}_{1} & \mathbf{0} & \mathbf{0}
\end{array}\right] \\
& \mathbf{T}_{2}=\left[\begin{array}{lll}
0 & 0 & 0 \\
\mathbf{0} & \mathbf{e}_{2} & \mathbf{0}
\end{array}\right] \\
& \mathbf{T}_{3}=\left[\begin{array}{lll}
0 & 0 & 0 \\
\mathbf{0} & \mathbf{0} & \mathbf{e}_{3}
\end{array}\right]
\end{aligned}
$$

The mapping from the active joint-rate array $\dot{\mathbf{u}}$ into the twist $\mathbf{t}_{j+3}$ of the $j$ th limb-link is not that straightforward. We do this in two steps. First, let us map the $j$ th limb-rate array $\dot{\gamma}_{j}$ into the $j$ th limb-link twist $\mathbf{t}_{j+3}$.

$$
\mathbf{t}_{j+3}=\dot{u}_{j}\left[\begin{array}{c}
0 \\
\mathbf{e}_{j}
\end{array}\right]+\dot{\varphi}_{A j}\left[\begin{array}{c}
1 \\
\left(l_{c} / l\right) \mathbf{E m}_{j}
\end{array}\right]=\mathbf{U}_{j+3} \dot{\gamma}_{j}
$$

with

$$
\mathbf{U}_{j+3}=\left[\begin{array}{ccc}
0 & 1 & 0 \\
\mathbf{e}_{j} & \left(l_{c} / l\right) \mathbf{E m}_{j} & \mathbf{0}
\end{array}\right] \quad j=1,2,3
$$

where $\mathbf{m}_{j}$ denotes the vector stemming from $A_{j}$ and ending at $M_{j}$. Moreover, $l$ denotes the length of the limb-link, $l_{c}$ the distance from $A_{j}$ to the c.o.m. of the limb-link. Then, according to (3) and (5), the mapping from the active jointrate array $\dot{\mathbf{u}}$ into the $j$ th limb-rate array $\dot{\gamma}_{j}$ is obtained:

$$
\dot{\gamma}_{j}=\mathbf{J}_{j}^{-1} \mathbf{H}^{-1} \mathbf{D} \dot{\mathbf{u}}, \quad j=1,2,3
$$

Therefore, the twist-shaping matrices $\mathbf{T}_{j+3}, j=1,2,3$, of the limb-links are

$$
\mathbf{T}_{j+3}=\mathbf{U}_{j+3} \mathbf{J}_{j}^{-1} \mathbf{H}^{-1} \mathbf{D}, \quad j=1,2,3
$$

The last, namely, the 7 th moving rigid body is the MP. Based on (5), its twist-shaping matrix is

$$
\mathbf{t}_{7}=\mathbf{t}_{M} \rightarrow \mathbf{T}_{7}=\mathbf{H}^{-1} \mathbf{D}
$$

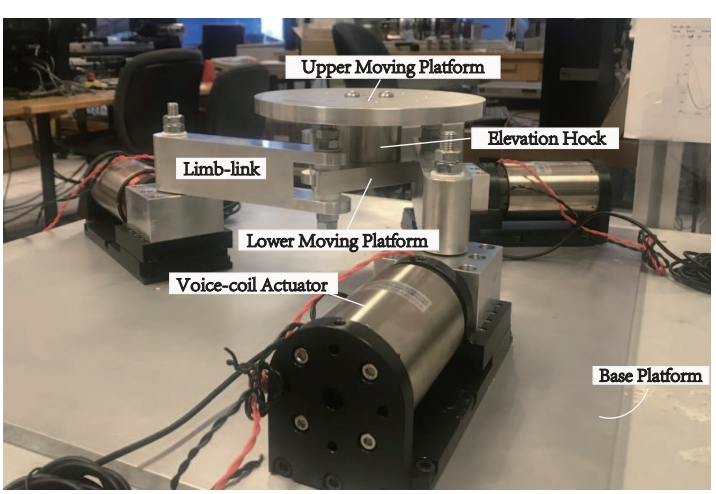

Fig. 3. Photo of the prototype of the 3-PRR planar parallel robot

\section{Model VALidation}

A desk-top scale prototype of the 3-PRR robot was designed and prototyped. Its prototype is shown in Fig. 3.

The robot is driven by three identical voice-coil actuators ${ }^{6}$. The slider of the actuated $\underline{P}$ joint contains the moving coil of the actuator and the plate attached to the moving coil. The MP is composed of three parts, namely, the lower moving platform, the elevation hock and the upper moving platform.

The side length $a$ of the MP triangle, the side length $b$ of the BP triangle and the length $l$ of the limb-link are given below:

$$
a=110 \mathrm{~mm}, \quad b=400 \mathrm{~mm}, \quad l=159.15 \mathrm{~mm}
$$

Moreover, the inertia properties of the different parts are recorded in Table I.

TABLE I

INERTIA PROPERTIES OF THE PARTS IN THE ROBOT

\begin{tabular}{|c|c|c|}
\hline Part & Mass $(\mathbf{g})$ & Moment of inertia ${ }^{\mathrm{a}} \mathbf{( g \cdot \mathbf { m m } ^ { \mathbf { 2 } } )}$ \\
\hline Actuator moving coil & 1020 & - \\
\hline Actuator plate & 449.66 & - \\
\hline Limb-link & 638.63 & 1578606.60 \\
\hline Lower moving platform & 417.35 & 716283.92 \\
\hline Elevation puck & 287.65 & 132353.74 \\
\hline Upper moving platform & 1047.57 & 5324476.16 \\
\hline
\end{tabular}

In order to validate the above-derived model, kinematics and dynamics simulation tests of the robot under different trajectories were conducted not only in Matlab, based on the mathematical models derived in Sections 3 and 4, but also in ADAMS, based on its CAD model. Two test trajectories were selected to represent various motions of the robot. These trajectories are expressed by the displacements of the MP centroid $C$, in the Cartesian frame, and the angle of rotation of the MP from its reference pose of Fig. 2. The trajectories are combinations of the high-frequency, small-amplitude harmonic

${ }^{6}$ The actuators in the 3-PRR planar robot are VCAR02620249-00A, manufactured by SUPT Motion (http://suptmotion.com/en/productshow_15_76.html). Their total stroke is $24.9 \mathrm{~mm}$, while their peak force is $26 \overline{2} \mathrm{~N}$. 
motions along $X$-, $Y$-axes and around $Z$-axis, as described below:

$$
\begin{aligned}
& \left\{\begin{array}{l}
x_{1}(t)=-2 \sin \left(20 \pi t+\frac{\pi}{6}\right)+1(\mathrm{~mm}) \\
y_{1}(t)=4 \sin \left(20 \pi t-\frac{\pi}{6}\right)+2(\mathrm{~mm}) \\
\theta_{1}(t)=(3 / 180) \pi \sin (20 \pi t)(\mathrm{rad})
\end{array}\right. \\
& \left\{\begin{array}{l}
x_{2}(t)=-2 \sin \left(20 \pi t+\frac{\pi}{6}\right)+1(\mathrm{~mm}) \\
y_{2}(t)=4 \sin \left(20 \pi t-\frac{\pi}{6}\right)+2(\mathrm{~mm}) \\
\theta_{2}(t)=(3 / 180) \pi \sin (10 \pi t)(\mathrm{rad})
\end{array}\right.
\end{aligned}
$$

Under Matlab, the simulation test trajectories are first transformed into the motor displacements in joint space via the inverse-kinematics model. Then, based on the inversedynamics model, the motor forces were calculated. Under ADAMS, the CAD model of the robot is shown in Fig. 4. In the two test trajectories, the motor displacements and forces calculated from the mathematical models and simulated in ADAMS, are shown in Figs. 5 and 6.

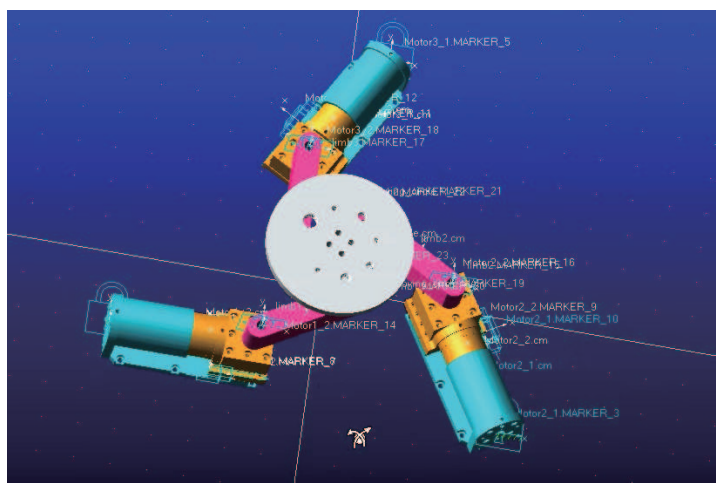

Fig. 4. CAD model of the 3-PRR planar parallel robot in ADAMS simulation

The results calculated via the mathematical models and those reported by ADAMS are apparently in agreement, which shows that the kinematics and the dynamics models derived in this paper, based on screw theory and the NOC, are correct. They can be used in the dynamics simulation and model-based control of the robot introduced in this paper.

\section{CONCLUSIONS}

A systematic approach to the formulation of the dynamics of PKMs, based on the concept of the NOC, was proposed. This approach was demonstrated on a planar parallel robot designed for high-frequency, small-amplitude displacements. Its kinematics and dynamics modeling were reported. Then, upon comparison with the ADAMS simulation results, our methodology is proven to be not only accurate, but also effective for dynamics modeling. It can be used for the dynamic simulation and real-time control of a parallel robot for high-frequency, small-amplitude displacements. Finally, the physical prototype of the robot introduced here is in its final stages of commissioning. Further progress on this project is the subject of a forthcoming paper.
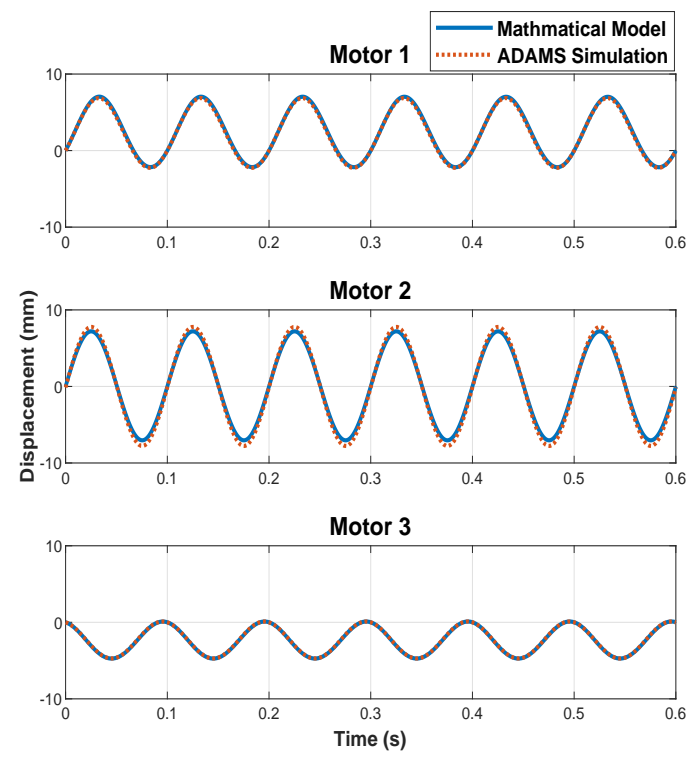

(a) Motor displacements
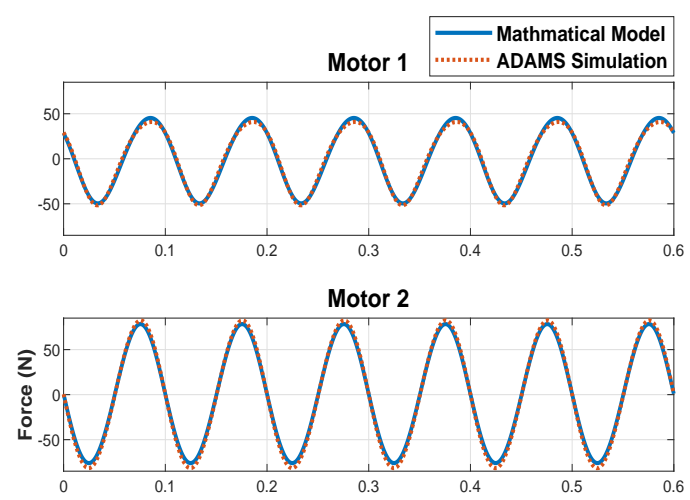

Motor 3

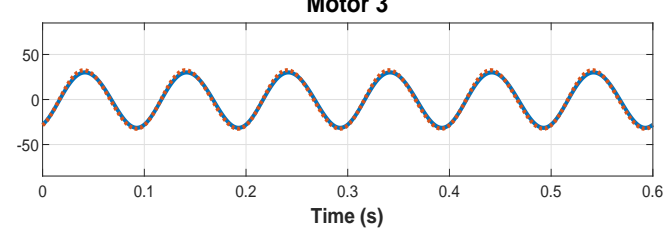

(b) Motor forces

Fig. 5. Mathematical model vs. ADAMS simulation under Trajectory 1

\section{ACKNOWLEDGMENT}

The first author would like to acknowledge the McGill Engineering Doctoral Award (MEDA) and the Chinese Scholarship Council (CSC) Scholarship (No. 201708880005) for their financial support. The second and third authors acknowledge the support received from Canada's Natural Sciences and Engineering Research Council (NSERC) through Grants No. RGPIN-2015-03864 and RGPIN-2016-04692, respectively. 

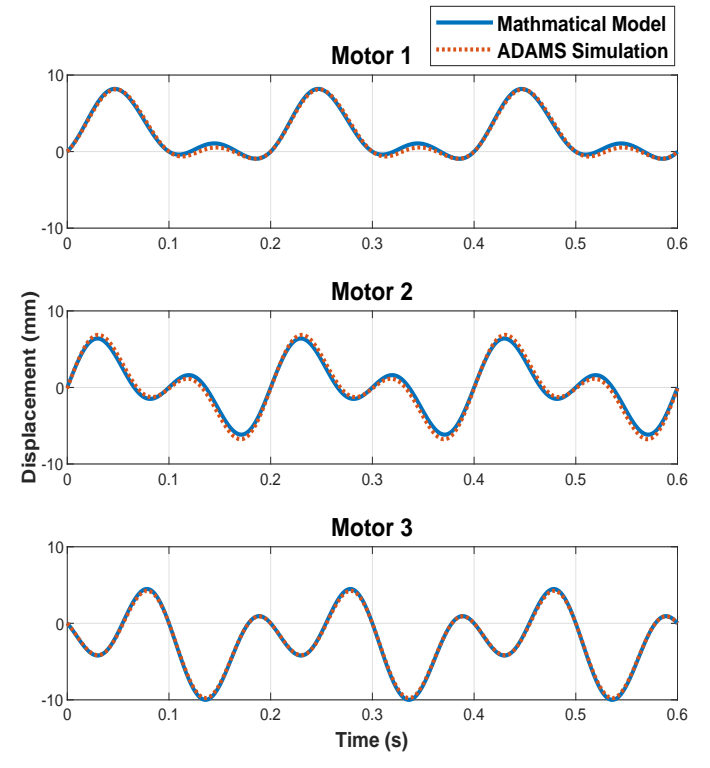

(a) Motor displacements
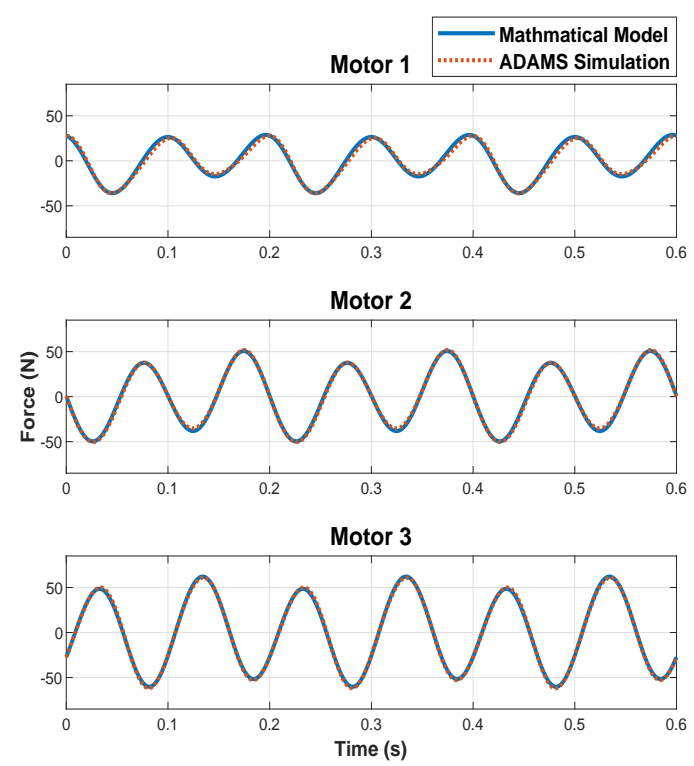

(b) Motor forces

Fig. 6. Mathematical model vs. ADAMS simulation under Trajectory 2

\section{APPENDICES}

\section{A. Inverse Displacement Analysis}

Here, we focus on the calculation of the joint variables, $\left\{\varphi_{A j}\right\}_{1}^{3}$ and $\left\{\varphi_{M j}\right\}_{1}^{3}$, given the robot posture $\mathbf{p}=$ $\left[\begin{array}{lll}\theta & x_{c} & y_{c}\end{array}\right]^{T}$.

Let $\mathcal{L}_{j}$ denote the line along the $j$ th BP side. This line is parallel to $\mathbf{e}_{j}$ and passes through $B_{j}$, whose coordinates in the $O X Y$ frame are

$$
\left[\begin{array}{l}
x_{B j} \\
y_{B j}
\end{array}\right]=R\left[\begin{array}{c}
\cos \left(\phi_{j}-\pi / 6\right) \\
\sin \left(\phi_{j}-\pi / 6\right)
\end{array}\right]
$$

where $R$ denotes the circumradius of the BP triangle. The equation of $\mathcal{L}_{j}$ in the $O X Y$ frame is thus expressed as

$$
y-y_{B j}=\tan \phi_{j}\left(x-x_{B j}\right), \quad j=1,2,3
$$

The distance $d_{j}^{C}$ from $C$ to $\mathcal{L}_{j}$ is calculated as

$$
d_{j}^{C}=\frac{\left|x_{c} \tan \phi_{j}-y_{c}+y_{B j}-x_{B j} \tan \phi_{j}\right|}{\sqrt{\tan ^{2} \phi_{j}+1}}, \quad j=1,2,3
$$

The distance $d_{j}^{M}$ from $M_{j}$ to $\mathcal{L}_{j}$ is, in turn,

$$
d_{j}^{M}=d_{j}^{C}-r \sin \left(\frac{\pi}{6}-\theta\right), \quad j=1,2,3
$$

where $r$ denotes the circumradius of the MP triangle. Therefore, the joint variables $\varphi_{A j}$ and $\varphi_{M j}$ are calculated as

$$
\varphi_{A j}=\arcsin \left(d_{j}^{M} / l\right), \quad \varphi_{M j}=\pi+\theta-\varphi_{A j}
$$

\section{B. Representation of the Twist-shaping Matrices}

When representing the twist-shaping matrices $\left\{\mathbf{T}_{i}\right\}_{1}^{6}$ in the $O X Y Z$ frame, vectors $\left\{\mathbf{e}_{j}\right\}_{1}^{3},\left\{\mathbf{p}_{M_{j}}\right\}_{1}^{3},\left\{\mathbf{m}_{j}\right\}_{1}^{3}$ and $\left\{\mathbf{p}_{A j}\right\}_{1}^{3}$ are needed. In the frame of interest, the foregoing vectors are expressed as

$$
\begin{gathered}
\mathbf{e}_{j}=\left[\begin{array}{c}
\cos \phi_{j} \\
\sin \phi_{j}
\end{array}\right], \mathbf{m}_{j}=\overrightarrow{A_{j} M_{j}}=l\left[\begin{array}{c}
\cos \left(\varphi_{A j}+\phi_{j}\right) \\
\sin \left(\varphi_{A j}+\phi_{j}\right)
\end{array}\right] \\
\mathbf{p}_{M j}=\overrightarrow{M_{j} C}=r\left[\begin{array}{c}
\cos \left(\varphi_{A j}+\varphi_{M j}-\pi / 6+\phi_{j}\right) \\
\sin \left(\varphi_{A j}+\varphi_{M j}-\pi / 6+\phi_{j}\right)
\end{array}\right] \\
\mathbf{p}_{A j}=\overrightarrow{A_{j} C}=\mathbf{m}_{j}+\mathbf{p}_{M j}
\end{gathered}
$$

\section{REFERENCES}

[1] B. Dasgupta and T.S. Mruthyunjaya. "A Newton-Euler formulation for the inverse dynamics of the Stewart platform manipulator." Mechanism and machine theory, 33(8), 1998, pp. 1135-1152.

[2] Z. Geng, L. S. Haynes, J.D. Lee, and R. L. Carroll, "On the dynamic model and kinematic analysis of a class of stewart platforms," Robotics and Autonomous Systems, vol. 9, 1992, pp. 237-254.

[3] A. Codourey and E. Burdet, "A body-oriented method for finding a linear form of the dynamic equation of fully parallel robots," IEEE International Conference on Robotics and Automation, vol. 2, 1997, pp. 1612-1618.

[4] K. Miller, "Optimal design and modeling of spatial parallel manipulators," The International Journal of Robotics Research, vol. 23, 2004, pp. $127-140$.

[5] C.M. Roithmayr and D.H. Hodges. Dynamics: Theory and Application of Kane's Method. Cambridge University Press, 2016.

[6] J. Angeles and S. Lee, "The formulation of dynamical equations of holonomic mechanical systems using a natural orthogonal complement," ASME Journal of Applied Mechanics, 55, 1988, pp. 243C244.

[7] J. Angeles and O. Ma, "Dynamic simulation of $\mathrm{N}$-axis serial robotic manipulators using a natural orthogonal complement." International Journal of Robotics Research, 7(5), 1988, pp. 32C47.

[8] J. Herv́e, 1999, "The Lie group of rigid body displacements, a fundamental tool for mechanism design," Mechanism and Machine Theory, vol. 34, pp. 719C730.

[9] R. von Mises, "Motorrechnung, ein neues Hilfsmittel der Mechanik, ZAMM-Journal of Applied Mathematics and Mechanics/Zeitschri ftür Angewandte Mathematik und Mechanik, 4(2), 1924, pp. 155C181.

[10] J. Angeles, "On Twist and Wrench Generators and Annihilators," in Seabra Pereira, M. and Ambrósio, J.C. (editors), Computer Aided Analysis of Rigid and Flexible Mechanical Systems, Kluwer Academic Publishers B. V., Dordrecht, 1994, pp. 379-411. 